\title{
Plantar Fascia Acute Rupture: A Rare Entity - Case Report
}

\author{
Nuno Reais*, Filipa Oliveira, Pedro Atilano, Carlos Queirós, João Teixeira and Marta Gomes \\ Department of Orthopedics and Traumatology, Portugal
}

*Corresponding author: Nuno Reais, Department of Orthopedics and Traumatology, Centro Hospitalar de Entre o Douro e Vouga, EPE, Aveiro, Portugal

\section{ARTICLE INFO}

Received: 幽 November 16, 2019

Published:

Citation: Nuno Reais, Filipa Oliveira, Pedro Atilano, Carlos Queirós, João Teixeira, Marta Gomes. Plantar Fascia Acute Rupture: A Rare Entity - Case Report. A Mini Review. Biomed J Sci \& Tech Res 23(2)-2019. BJSTR. MS.ID.003882.

\begin{abstract}
Plantar fascia acute rupture is a rare clinical entity, with very few existing reports in the literature. The described cases occur mainly in athletes, during an intense physical effort. Its diagnosis is mainly clinical; however, it is possible nowadays to use complementary means of diagnosis to help us such as ultrasound or MRI. Conservative treatment is, historically, the most used and recommended treatment, with favorable results. Although the role for surgical treatment is not well established yet, this is usually reserved for cases that are refractory to conservative treatment. The authors present a case of an athlete who sustained an acute plantar fascia rupture, that was submitted to conservative treatment with a good clinical outcome, being able to return regular physical activity with no limitations after the treatment period.
\end{abstract}

Keywords: Plantar Fascia; Acute Rupture

\section{Introduction}

The plantar fascia is one of the most important structures support of the plantar arch. While plantar fasciitis is a well-known and common condition, plantar fascia rupture is rare and may occur spontaneously during physical activity. Plantar fascia acute rupture was first described by Leach et al. [1,2]. It is mainly described in athletes, and there are a few known factors that predispose to its occurrence, such as prior corticosteroid use, and plantar fasciitis [3-5]. The patients usually describe a tearing sensation with intense pain of the sole of the foot. So, previous plantar fasciitis, previous corticosteroid injection and acute onset of symptoms during athletic activity are three factors that are important to keep in mind in making the diagnosis. The cases described in the literature are scarce and there is no consensus on the best therapeutic strategy to adopt. The mainstay of treatment is based on conservative measures such as rest, physiotherapy, cryotherapy and analgesia. Surgical treatment is only recommended if conservative treatment fails. The main purpose of this article is to describe the clinical and imagiologic diagnosis of this entity.

\section{Case Report}

The authors describe the case of a 30 years-old male athlete who presented to the Emergency Department with acute right foot plantar pain suddenly triggered by a sprint. He presented with gait limitation, and inability to do weightbearing in that foot. He had history of previous fasciitis, but only of the contralateral foot. He did not have any histoy of corticosteroid injection. Physical examination showed pain at palpation of the sole, and plantar edema and ecchymosis (Figures 1A-1D), as well as bilateral pes cavus (Figures 2A \& 2B). The patient performed an X-ray in the emergency department, which showed no signs of osseous lesions. Magnetic resonance imaging of the foot was performed the day after the traumatic event, which revealed rupture of the plantar fascia with edema of the surrounding tissues (Figures 3A \& 3B). After clinical and imagiological confirmation of the diagnosis the patient underwent conservative treatment. The treatment consisted of 3 weeks of non-weightbearing with a short leg removable cast-boot. 


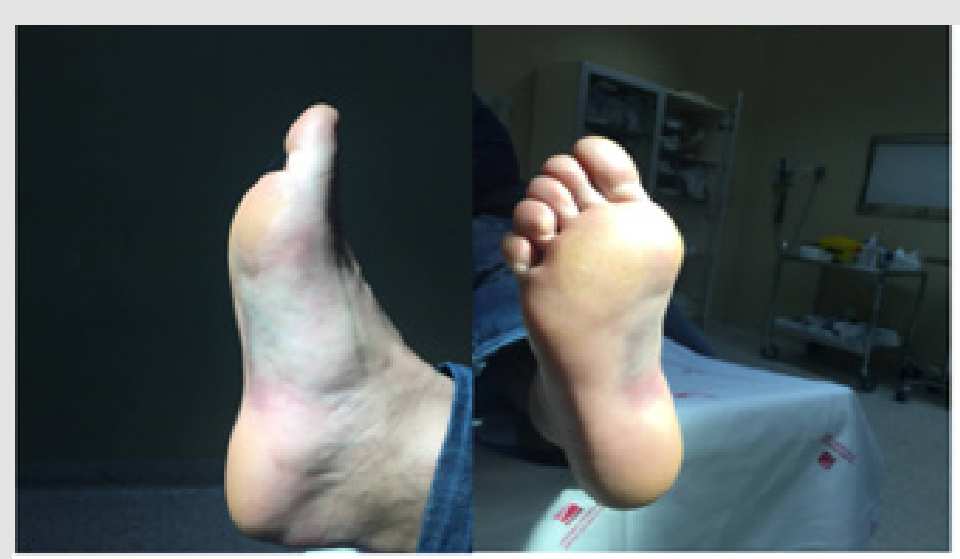

A

B

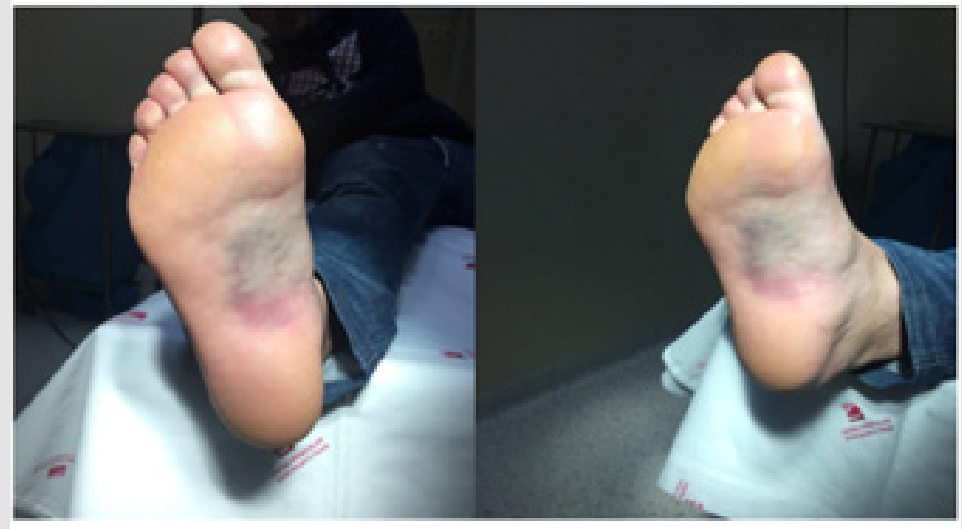

C

D

Figures 1A-1D: In the ER the patient presented with pain, plantar edema and equimosis.

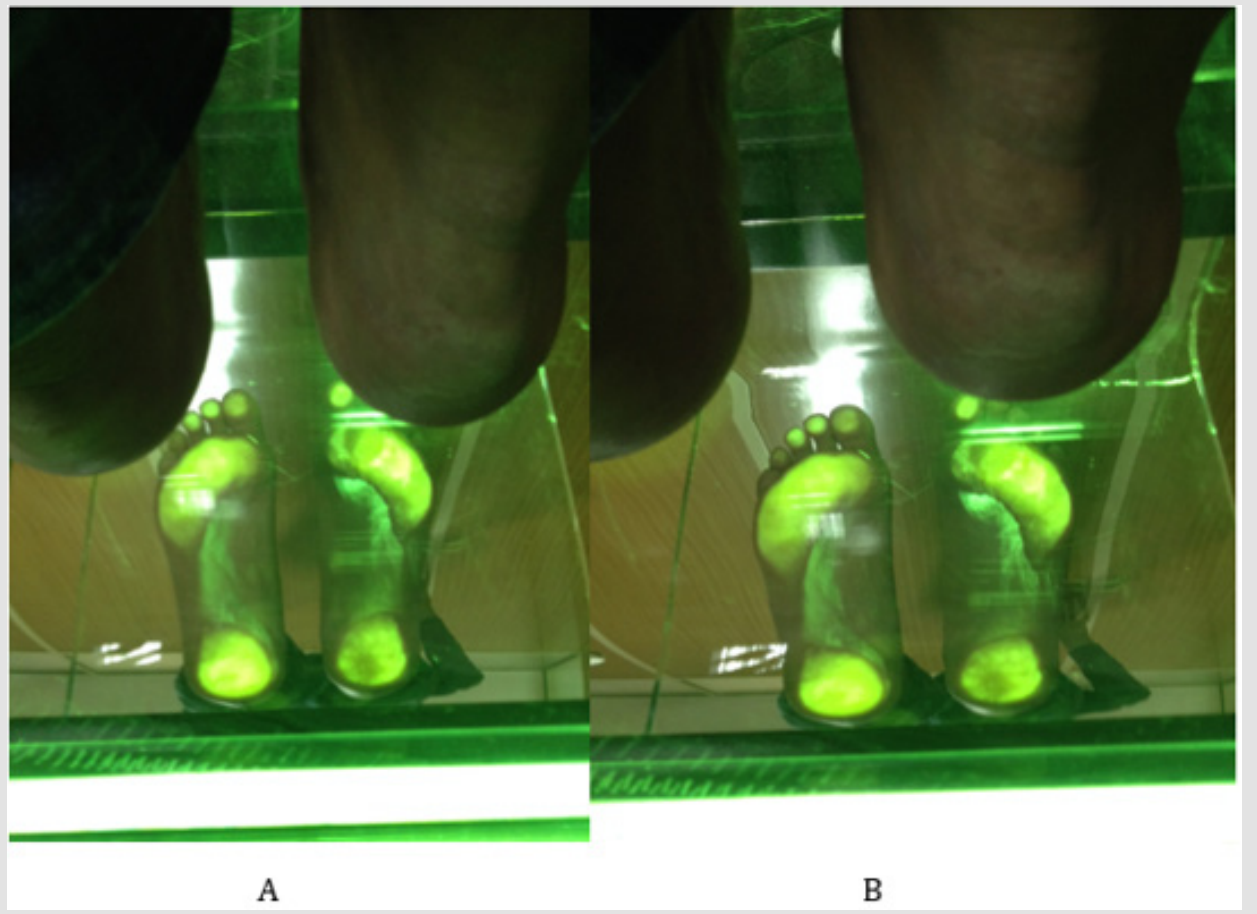

Figures 2A \& 2B: The patient presented bilateral pes cavus as seen in the podoscope. 


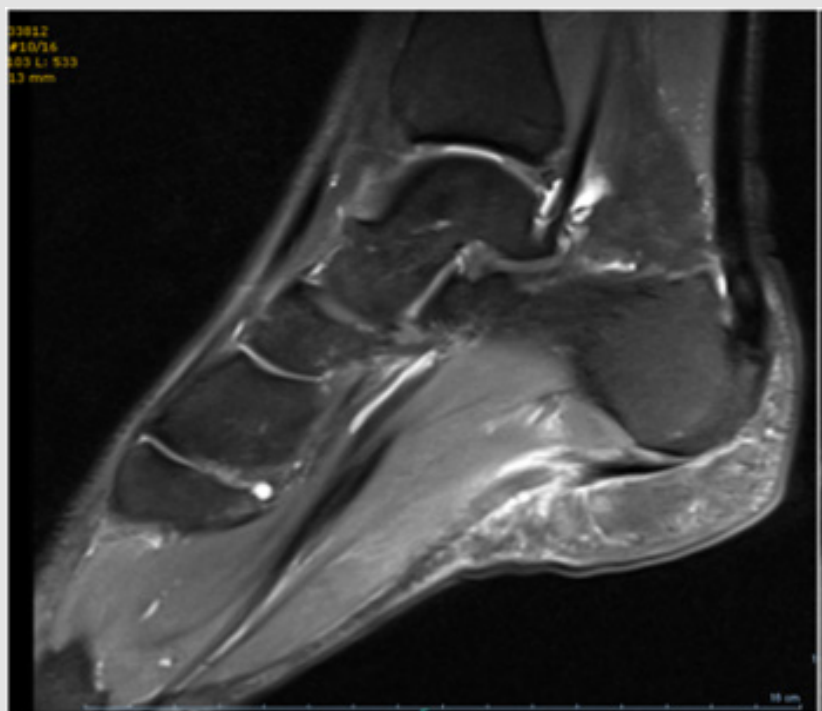

A

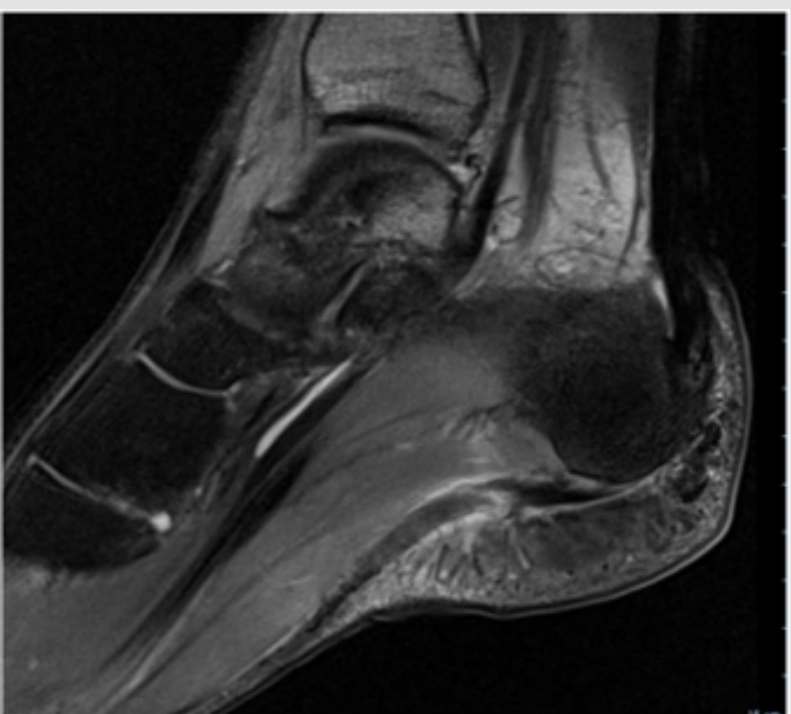

B

Figures 3A \& 3B: Sagital MRI of the patient showing the plantar fascia rupture and edema of the surrounding tissue.

Protected weightbearing was then initiated with a Walker Boot, as tolerated by the patient. Physical therapy was initiated 1 week after the injury with electrical stimulation, pulsed ultrasound, manual deep tissue massage, and gentle stretching. NSAID's and cryotherapy were started in the day of the injury and for a period of 2 weeks; after these two weeks, only if the patient had pain. After 6 weeks the patient was able to walk with full weightbear and no limitation of the gait and had no need of using the Walker Boot. After 2 months the patient could return to physical activity and presented no limitations. The patient was recommended to use semiflexible polypropylene orthoses tailormade according to its neutral subtalar position.

\section{Discussion}

There are a few factors described as known to predispose to plantar fascia rupture: Plantar fasciitis is one of the most common diseases in the athletes. The mechanism of acute injury to the plantar fascia may be due to an excessive overload on the weakened structure. Rupture could represent an acute-on-chronic injury as well, particularly in runners $[1,5,6]$. The presented patient had history of plantar fasciitis; however, it was only of the contralateral foot. He had never had previous pain symptoms of the right. Corticosteroid injection is frequently used in the treatment of plantar fasciitis. It is believed that it can accelerate the weakening of the tissues of the already diseased plantar fascia; In previous reports, an association has been shown between plantar fascia rupture and corticosteroid injection $[3,4]$. Despite having contralateral plantar fasciitis, this patient had never done any corticosteroid injection to treat it. The treatment of plantar fasciitis had only consisted of rest, ice and physiotherapy. He also claims that he had never done oral corticotherapy for any other reason. Pes planus/Pes cavus- Previous studies have reported altered biomechanics in high-arched and low-arched feet. High-arched feet do not have the necessary mobility to absorb ground reaction forces. Because of being unable to dissipate forces from heel strike to midstance, the load applied to the plantar fascia is highly increased. The opposite mechanism occurs with pes planus. Whether due to the consequent excessive or too little motion of the foot (pes planus and pes cavus respectively), that these conditions cause, they are both risk factors to the development of plantar fasciitis and, consequently, plantar fascia rupture [6]. The presented patient had pes cavus which may explain a certain degree of predisposition to this lesion. The diagnosis is mainly clinical. The patient presented a typical history of sudden "pop" of the heel and acute onset of pain after an abrupt effort. The authors highlight the importance of clinical history and physical examination. A high index of clinical suspicion is required for the diagnosis of this lesion. Ultrasound and MRI may help confirming the diagnosis. In this context MRI constitutes the Gold Standard for diagnosis of this lesion [7]. The mainstay of treatment is conservative treatment with a positive clinical outcome for most cases. Our patient was able to resume its regular physical activity after 2 months of conservative treatment. Only in the rare cases that don't improve after conservative treatment, should the surgical treatment be considered [1].

\section{Conclusion}

Acute plantar fascia rupture is a rare entity whose diagnosis is based primarily on clinical parameters (clinical history and physical examination) and may be complemented by complementary imaging techniques (of which the MRI is the Gold-Standard). In most cases recovery is complete after conservative treatment, being the surgical treatment considered only in refractory cases. 


\section{References}

1. Leach R, Jones R, Silva T (1978) Rupture of the plantar fascia in athletes. J Bone Joint Surg Am 60(4): 537-539.

2. Rolf C, Gunter P, Ericsäter J, Turan I (1997) Plantar fascia rupture: diagnosis and treatment. J Foot Ankle Surg 36(2): 112-114.

3. Sellman JR (1994) Plantar fascia rupture associated with corticosteroid injection. Foot Ankle Int 15(7): 376-381.

4. Acevedo JI, Beskin JL (1998) Complications of plantar fascia rupture associated with corticosteroid injection. Foot Ankle Int 19(2): 91-97.

ISSN: 2574-1241

DOI: 10.26717/BJSTR.2019.23.003882

Nuno Reais. Biomed J Sci \& Tech Res

(C) This work is licensed under Creative Submission Link: https://biomedres.us/submit-manuscript.php
5. Saxena A, Fullem B (2004) Plantar fascia ruptures in athletes. Am J Sports Med 32(3): 662-665.

6. Bolgla L, Malone T (2004) Plantar Fasciitis and the windlass mechanism: a biomechanical link to clinical practice. J Athl Train 39(1): 77-82

7. Draghi F, Gitto S, Bortolotto C, Draghi AG, Ori Belometti G (2017) Imaging of plantar fascia disorders: findings on plain radiography, ultrasound and magnetic resonance imaging. Insights Imaging 8(1): 69-78.

\section{Assets of Publishing with us}

- Global archiving of articles

- Immediate, unrestricted online access

- Rigorous Peer Review Process

- Authors Retain Copyrights

- Unique DOI for all articles

https://biomedres.us/ 